\title{
HISTOCHEMICAL STUDY OF THE SEMINAL VESICLE SECRETION OF THE INDIAN HEDGEHOG WITH PARTICULAR REFERENCE TO THE CRYSTALLOID BODIES
}

\author{
PUSHPAMALA P. BIDWAI AND S. R. BAWA \\ Department of Biophysics, Panjab University, Chandigarh, India
}

(Received 25th Fune 1971, accepted 25th October 1971)

\begin{abstract}
Summary. During the breeding season, the seminal vesicles of the Indian hedgehog secrete an amorphous material and crystalloid bodies. The latter are absent in the non-rutting period. The amorphous secretion of the rutting, as well as of the non-rutting, seminal vesicles show identical histochemical reactions in containing mucoproteins, mucopolysaccharides and appreciable amounts of alkaline phosphatase. The crystalloid bodies are complexes of glycolipids and mucoproteins and, unlike the amorphous secretion, they show an intense acid phosphatase activity. Although progressive development of the crystalloid material in the apical cells of the seminal vesicle epithelium has not been seen, there is similarity of these cells to the crystalloid bodies located in the lumen. The secretion of these crystalloids seems to be dependent upon the hormonal status of the animal. While they are present in the seminal vesicles of hedgehogs treated with male goat pituitary extracts in the non-breeding season, they cannot be demonstrated in the seminal vesicles of a control group.
\end{abstract}

\section{INTRODUCTION}

The Indian hedgehog, Paraechinus micropus, is sexually active between late April and late September; thereafter, its testes and associated sex glands abruptly retrogress and remain quiescent throughout the winter. The insectivores are unique in that crystalloid bodies are present in their seminal vesicles (Mann, 1964) and prostate glands (Eadie, 1948a, b). Biochemical (Hopkins, 1911; Mann, 1964) and histomorphological studies (Marshall, 1911; Allanson, 1934; Young, 1961) of the seminal vesicles of European hedgehog, Erinaceous europeas, have been published. Several reports are available on the histochemistry of the seminal vesicles of other mammals, both seasonal and continuous breeders (Wislocki, 1949; Seaman, 1956; Aitken, 1960; Aughey, 1969; Riva \& Stockwell, 1969), but so far there appears to have been no attempt to study the histochemistry of the seminal vesicles of hedgehogs. In this communication, we present a histochemical study of the amorphous secretion and the crystalloid bodies in the rutting and the non-rutting seminal vesicles of the 
Indian hedgehog. In addition, we have also studied the probable origin of these bodies and their dependence on the hormonal status of the hedgehogs.

\section{MATERIAL AND METHODS}

Twenty-five hedgehogs collected from Rajasthan between August 1968 and September 1969 were used in this study. The animals were anaesthetized with chloroform and the seminal vesicles were dissected out. Small pieces of the material were fixed in a variety of chemical fixatives, i.e. Bouin's, Zenker, Carnoy, Lewistsky saline, Helly's, Regaud, 10\% neutral formalin, formolcalcium and cold acetone. The tissue blocks were subsequently washed, dehydrated and embedded in paraffin-wax and sections were cut at 5 to $10 \mu \mathrm{m}$. The formol-calcium-fixed tissues were post-fixed in Baker's dichromate calcium and after thorough washing were embedded in gelatin and the sections were cut at 7 to $10 \mu \mathrm{m}$ on a cold microtome. Routine haematoxylin-eosin, ironhaematoxylin and trichrome staining was performed according to the techniques described by Humason (1962).

Histochemical localization of nucleic acids, proteins, polysaccharides, lipids and acid and alkaline phosphatases was achieved according to the techniques cited by Pearse (1961). In order to demonstrate the influence of hormones on the secretion of the seminal vesicles, ten hedgehogs, weighing approximately $300 \mathrm{~g}$ each, whose seminal vesicles were in a retrogressed state, were selected. They were allotted to experimental and control groups of five animals each. Over a period of 4 weeks, from the 2nd week of January to the 2nd week of February 1971, every animal from the experimental group was given a weekly intraperitoneal injection of $40 \mathrm{mg}$ of a suspension of fresh pituitary tissue in $1 \mathrm{ml}$ normal saline. The control animals received an intraperitoneal injection of an equal amount of normal saline only. The pituitary extract was prepared fresh from male goat pituitaries according to the method described by Carrol \& Gregory (1962). The hedgehogs were killed 1 week after the administration of the last injection. The testis tubules of the control animals were examined immediately under the phase contrast microscope to check that these animals were in the sexually inactive phase. The seminal vesicles of the hedgehogs in both groups were fixed in Bouin's and Zenker and were subsequently processed separately for paraffin-wax embedding. The sections were cut at 7 to $10 \mu \mathrm{m}$ and stained with PAS and haematoxylin.

\section{RESULTS AND DISCUSSION}

The Indian hedgehog, like other seasonal breeders (Mossman, John, Lawlah

EXPLANATION OF PLATE 1

Fig. 1. PAS-positive crystalloids and amorphous secretion in the cavity of the acini of Indian hedgehog, Paraechinus micropus, seminal vesicle. PAS $\times 85$.

Fig. 2. Intense acid phosphatase activity in the crystalloid bodies-rutting hedgehog, seminal vesicle. Gomori technique $\times 85$.

Fig. 3. Large number of crystalloids seen in the seminal vesicle of a hedgehog treated with pituitary extract. PAS $\times 100$.

Fra. 4. Seminal vesicle of the control hedgehog lacking the crystalloid bodies. PAS $\times 100$. 
PLATE 1

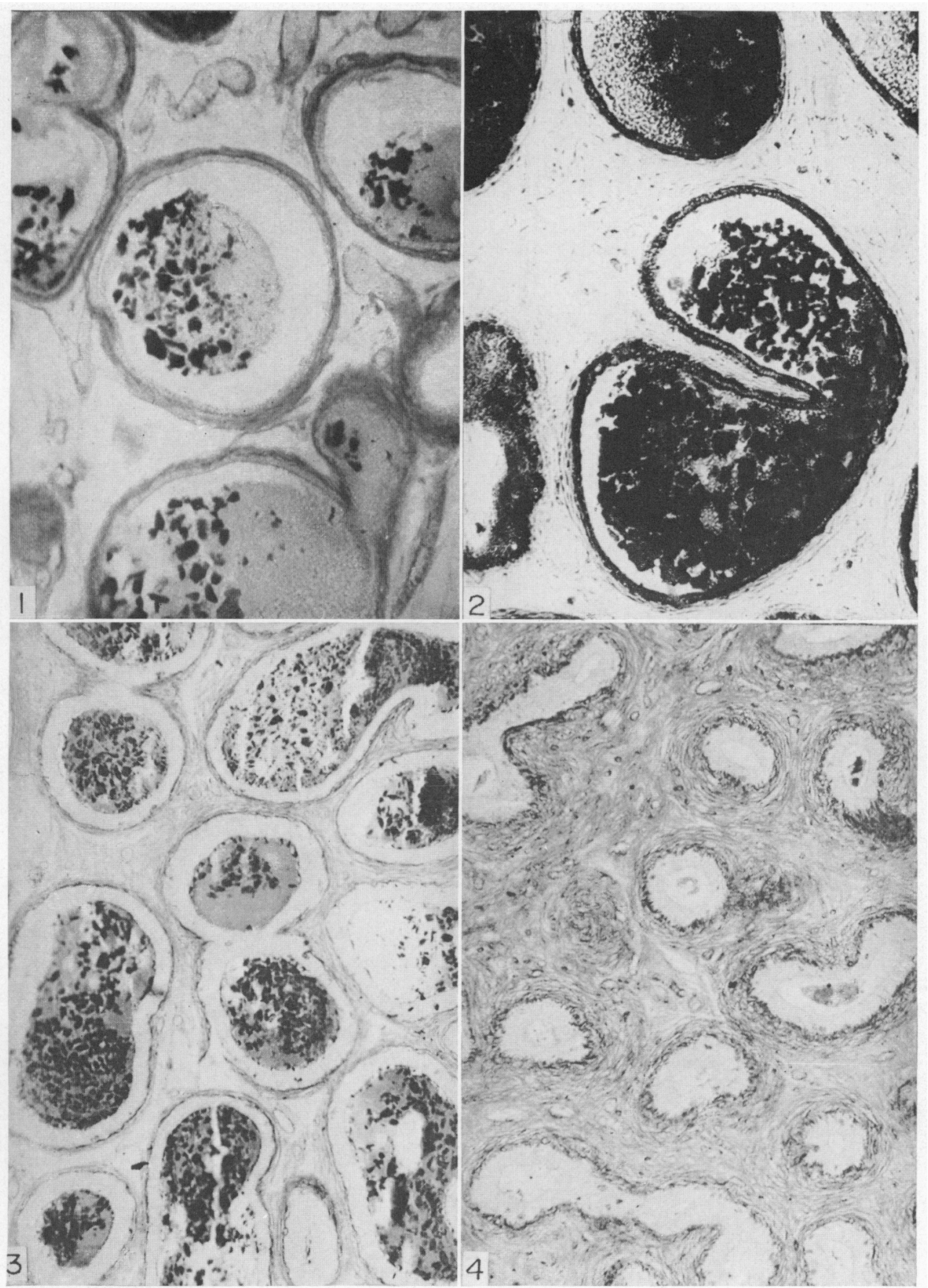

(Facing p. 220) 
\& Bradley, 1932; Allanson, 1934; Mann, 1964), shows regression of the seminal vesicles in the non-breeding season when the secretory activity is at its lowest ebb. By contrast, at the beginning of the breeding season, the seminal vesicle epithelium shows intense secretory activity, the secretion consisting of an amorphous material and crystalloid bodies. Although the acini of the seminal vesicles in the non-rutting period are reduced in size, they contain an appreciable amount of secretion but the crystalloid bodies are conspicuous by their absence. These bodies are distinctly visible in the lumen of the seminal vesicles of the sexually active hedgehog.

\section{Amorphous secretion}

The amorphous secretion in the seminal vesicles of hedgehogs in the breeding and non-breeding seasons displays identical histochemical reactions. The secretion colours moderately with eosin, haematoxylin and trichrome stains. The negative reactions of methyl green-pyronin, chrome alum-haematoxylin and Feulgen suggests that the amorphous secretion is devoid of DNA and RNA. The positive reaction (Pl. 1, Fig. 1) with periodic acid-Schiff (PAS) indicates the presence of polysaccharides, but the deficiency or absence of glycogen from this secretion is shown by the negative reaction with Best's carmine and also the resistance to diastase digestion before PAS staining. The polysaccharides which show a negative reaction to Alcian blue stain moderately with Mayer's mucicarmine implying that the secretory material contains mucoproteins and mucopolysaccharides. The protein component of the secretion is indicated by the positive-mercury bromophenol blue and Ninhydrin Schiff tests. The presence of SH- and SS-groups in the proteins is suggested by an intense positive ferric-ferricyanide reaction. The absence of the lipids in the amorphous secretion is confirmed by the negative Sudan Black B and other fat localizing tests. The secretion lacks acid phosphatase activity (Pl. 1, Fig. 2) but shows intense alkaline phosphatase reaction by Gomori's method.

\section{Crystalloid bodies}

The crystalloid bodies, irregular in shape and of variable size, stain with eosin haematoxylin, acid and basic fuchsin and Masson's and Heidenhain's trichrome stains. They contain polysaccharides and stain intensely with PAS (Pl. 1, Fig. 1). The glycogen of these bodies colours moderately with Best's carmine and there is very little effect of diastase incubation on their ability to stain with PAS. The bodies stain moderately with Mayer's mucicarmine but a negative reaction with Alcian blue suggests the absence of acid mucopolysaccharides. Similarly, a negative reaction with Feulgen and other nucleic acid stains indicates the absence of DNA and RNA. A few of the crystalloid bodies in the lumen of the seminal vesicle acini reveal intact nuclei. These bodies contain proteins as shown by the positive mercury bromophenol blue and Ninhydrin Schiff reactions. Intense blue colouration with ferric ferricyanide suggests the presence of SS- and SH-groups. The ability of the crystalloid bodies to stain positively with Sudan black B, Oil red O, Nile blue sulphate and acid haematin implies the presence of neutral and acidic lipids, the latter containing phospholipids. These crystalloid bodies, unlike the amorphous 
secretion, show intense acid phosphatase activity (Pl. 1, Fig. 2). The histochemical reactions of the crystalloid bodies, which are distinctly identifiable in the lumen of the seminal vesicles of the sexually active hedgehogs, indicate that the bodies are complexes containing mucoproteins and lipids and that they possess intense acid phosphatase activity.

As in other mammals, the seminal vesicle epithelium of the sexually active hedgehog contains two types of cell, basal cells and apical cells. Some of the apical cells in a few of the glandular acini stain diffusely with PAS and share other histochemical staining properties with those of the crystalloid bodies in the lumen. However, we have failed to observe a progressive accumulation of the crystalloid material in the epithelial cell cytoplasm, which appears to be uniformly stained. Such diffusely stained PAS-positive cells are usually observed in masses. These cells are probably desquamated into the lumen of the glandular acini. Such desquamation of the apical cells of the seminal vesicle epithelium may account for the presence of some of the nuclei observed in the luminal crystalloid bodies. The apical cells showing a similarity in staining with that of the crystalloid bodies are very few compared with the large number of crystalloid bodies observed in the lumen of all the acini studied by us. These observations suggest that the apical cells probably acquire their PAS-staining capacity just before desquamation. This explanation can account for the occurrence of the PAS-stained cells in only a few of the acini, which are in close proximity to each other, whereas the crystalloids are found in abundance in all the acinar lumina.

The seminal vesicles of those hedgehogs which were treated with pituitary extract in the non-breeding season showed the presence of a large number of crystalloid bodies (Pl. 1, Fig. 3). These bodies were similar to those observed in the seminal vesicles of the sexually active normal hedgehogs. The control hedgehogs, however, failed to show such crystalloid bodies in their seminal vesicles (Pl. 1, Fig. 4). The retrogressed status of the seminal vesicles of the control hedgehogs was confirmed by the absence of live spermatozoa in their seminiferous tubules examined with phase optics. The experimental restoration of the secretion of the crystalloid bodies in the seminal vesicles of hedgehogs even in the non-breeding season, following the administration of gonadotrophic hormones, indicates that the formation and release of these crystalloid bodies may be hormone dependent.

\section{REFERENCES}

ArtKen, R. N. C. (1960) A histochemical study of the accessory glands of the boar. F. Anat. 94, 130.

Allanson, M. (1934) Reproductive processes of certain mammals. VII. Seasonal variation in the reproductive organs of the male hedgehog. Phil. Tran. $R$. Soc. 223, 277.

Aughey, E. (1969) Histology and histochemistry of the male accessory glands of the red deer, Cervus elaphus L. F. Reprod. Fert. 18, 399.

CARrol, F. D. \& Gregory, P. W. (1962) Responses of the Snell dwarf mouse to pituitary tissue from a bovine dwarf mutant. Proc. Soc. exp. Biol. Med. 109, 35.

EAdIE, W. R. (1948a) The male accessory reproductive glands of Condylura with notes on the unique prostatic secretion. Anat. Rec. 101, 59.

EADIE, W. R. (1948b) Corpora amylacea in the prostatic secretions and experiments on the formation of a copulatory plug in some insectivores. Anat. Rec. 102, 259.

Hopkins, F. G. (1911) Addendum: A note on the chemistry of the vesicular fluid of hedgehog. $\mathcal{F}$. Physiol., Lond. 43, 259. 
Humason, G. L. (1962) Animal tissue techniques. Freeman, San Francisco.

ManN, T. (1964) The biochemistry of semen and of the male reproductive tract. Methuen, London.

Marshall, F. H. A. (1911) The male generative cycle in the hedgehog with experiments on the functional correlation between the essential and accessory sexual organs. F. Physiol., Lond. 43, 246.

Mossman, H. W., John, W. Lawlah \& J. A. Bradley (1932). The male reproductive tract of the Sciuridae. Am. F. Anat. 51, 89.

Pearse, A. G. E. (1961) Histochemistry, theoretical and applied. Little, Brown and Company, Boston.

Rrva, A. \& Stockwell, R. A. (1969) A histochemical study of the human seminal vesicle epithelium. J. Anat. 104, 253.

Seaman, A. (1956) Cytochemical observations on corpora amylaceae. F. Urol. 76, 99.

Wrssocki, G. B. (1949) Seasonal changes in the testes, epididymides and seminal vesicles of deer investigated by histochemical methods. Endocrinology, 44, 167

Young, W. G. (1961) Sex and internal secretions, Vol. 1. Williams \& Wilkins, Baltimore. 